\title{
Intravenous immunoglobulin-associated hemolysis: risk factors, challenges, and solutions
}

This article was published in the following Dove Press journal:

International Journal of Clinical Transfusion Medicine

21 October 2016

Number of times this article has been viewed

\author{
Muhajir Mohamed ${ }^{1,2}$ \\ 'Department of Haematology, \\ Launceston General Hospital, \\ ${ }^{2}$ Launceston Clinical School, \\ University of Tasmania, Launceston, \\ Tasmania, Australia
}

Correspondence: Muhajir Mohamed Department of Haematology, Launceston General Hospital, Charles Street, Launceston, Tasmania, Australia

Tel +61367776688

Fax +6I 363487695

Email muhajirbm@yahoo.com

\begin{abstract}
Clinically significant hemolysis associated with intravenous immunoglobulin (IVIG) administration has recently been identified as a major problem requiring special attention, after increased incidence has been documented by US FDA since 2011. IVIG-associated hemolysis remains an under-recognized complication of IVIG therapy, and hence the exact incidence of hemolytic anemia induced by high dose IVIG is not clear. Patients at high risk of IVIG-associated hemolysis are non-O blood group recipients who receive high-dose IVIG for inflammatory or autoimmune disorder. Due to the immunomodulatory potential of high-dose IVIG, the clinical indications have increased over time. The objective of this article is to discuss the epidemiology, risk factors, and challenges of IVIG-associated hemolysis. Strategies to reduce the incidence of clinically significant hemolysis in patients treated with IVIG products and potential avenues for further research in this field are also discussed.
\end{abstract}

Keywords: IVIG, immunoglobulins, hemolysis, ABO, isoagglutinins, hemolytic anemia

\section{Background}

Intravenous immunoglobulin (IVIG) is prepared from large pools of plasma obtained from thousands of donors. IVIG contains predominantly immunoglobulin $\mathrm{G}$ (IgG) with small quantities of IgA and IgM. ${ }^{1}$ IVIG has clinical utility for a variety of hematological, neurological, immunological, and rheumatologic diseases. It is used as replacement therapy in cases of primary and secondary immune deficiency disorders and exhibits immunomodulatory effect in immune disorders such as idiopathic thrombocytopenic purpura, Guillain-Barre syndrome, myasthenia gravis, and chronic inflammatory demyelinating polyneuropathy. The clinical indications of IVIG which have been licensed by the United States Food and Drug Administration (FDA) are outlined in Table $1 .{ }^{2}$

IVIG is generally well tolerated. However, a small proportion of patients $(<5 \%)$ may experience adverse effects such as headache, backache, fever, chills, and rigors during infusions. These can be managed by reducing the rate of infusion and/or administration of analgesics. Some adverse effects are seen mainly with the use of high-dose IVIG such as thrombosis, aseptic meningitis, hemolysis, and renal failure. Viral transmission by IVIG products is an uncommon complication nowadays due to rigorous donor screening measures and addition of stringent antiviral steps in the manufacturing process. ${ }^{1}$

Hemolytic anemia is caused by the presence of blood group antibodies, namely, anti-A anti-B in IVIG products, ${ }^{3}$ and is an under-recognized complication of IVIG therapy. Hence, the exact incidence of hemolytic anemia induced by high-dose IVIG 
Table I The FDA-approved indications for IVIG

\begin{tabular}{ll}
\hline Diseases & Indications \\
\hline $\begin{array}{l}\text { I. Primary humoral } \\
\text { dimmunodeficiency }\end{array}$ & $\begin{array}{l}\text { For replacement therapy of primary } \\
\text { immunodeficiency disorders and to increase the } \\
\text { circulating antibody levels in patients with severe } \\
\text { impairment of antibody-forming capacity }\end{array}$ \\
2. ITP & $\begin{array}{l}\text { For rapid increase in platelet count to prevent } \\
\text { bleeding, control bleeding, or both } \\
\text { To increase the platelet count in a patient with }\end{array}$ \\
3. KD & $\begin{array}{l}\text { For the prevention of coronary artery aneurysms } \\
\text { associated with KD } \\
\text { In patients with acquired hypogammaglobulinemia, }\end{array}$ \\
4. B-cell CLL & $\begin{array}{l}\text { associated with B-cell CLL, for the treatment of } \\
\text { bacterial infections, and prevention of recurrent } \\
\text { bacterial infections } \\
\text { Bone marrow transplant recipients } \geq 20 \text { years } \\
\text { of age to reduce the risk of serious and other } \\
\text { infections, interstitial pneumonia, and acute graft } \\
\text { versus host disease in the first I00 days after } \\
\text { transplantation } \\
\text { In pediatric patients with HIV infection to } \\
\text { decrease the frequency of bacterial infections and } \\
\text { the frequency of hospitalizations }\end{array}$ \\
\hline
\end{tabular}

Abbreviations: CLL, chronic lymphocytic leukemia; FDA, Food and Drug Administration; HIV, human immunodeficiency virus; ITP, immune thrombocytopenic purpura; IVIG, intravenous immunoglobulin; KD, Kawasaki disease.

is uncertain. $\mathrm{ABO}$ antibodies or isoagglutinins are normally present in donors of $\mathrm{A}, \mathrm{B}$, and $\mathrm{O}$ blood groups used for IVIG production, with the exception of group $A B$ donors. Since $O$ and A blood groups together form $\sim 70 \%-80 \%$ of the world population, most of the plasma used in IVIG production is from these donors. Hence, IVIG will contain blood group antibodies that are likely to react with individuals of blood groups A, B, and AB. In such individuals, IVIG administration can potentially lead to hemolytic transfusion reactions (HTRs) similar to a blood transfusion mismatch. ${ }^{4}$ It has been observed that low-dose IVIG or subcutaneous immunoglobulin used in replacement therapy of primary and secondary immunodeficiency disorders is generally safe with a very low risk of hemolysis. ${ }^{5}$ However, due to the immunomodulatory potential of high-dose IVIG, the clinical indications and usage of high-dose IVIG have increased over time. ${ }^{6}$

The incidence of IVIG-associated hemolysis is $\sim 1$ per 1000 IVIG treatment episodes, with most occurring within $48 \mathrm{~h}$ of exposure. ${ }^{7}$ Majority of such hemolytic episodes are unnoticed or self-limiting with positive direct antiglobulin test (DAT) and mild decrease in hemoglobin ( $\mathrm{Hb}){ }^{8}$ Occasionally, hemolysis may lead to a clinically significant drop in $\mathrm{Hb}$, with severe cases requiring packed red blood cell (RBC) transfusion. ${ }^{9}$ Since 2011, the US FDA has received increased reports of HTRs after IVIG treatment from health care providers. ${ }^{10}$
The IVIG Hemolysis Pharmacovigilance Group definition of IVIG-associated hemolysis is a hemolytic episode occurring within 10 days of administration of IVIG as evidenced by the following criteria.

\section{Inclusion criteria}

A decrease in $\mathrm{Hb}$ of $\leq 10 \mathrm{~g} / \mathrm{L}$ and a positive DAT plus at least two of the following:

1. elevated reticulocyte count,

2. elevated serum lactate dehydrogenase,

3. elevated serum unconjugated bilirubin,

4. low serum haptoglobin,

5. hemoglobinemia (Figure 1),

6. hemoglobinuria, and/or

7. spherocytosis (Figure 2).

\section{Exclusion criteria}

1. Other causes of anemia such as
a. blood loss,
b. other drugs causing hemolysis, and
c. other diseases causing hemolysis,

2. negative DAT, and

3. no evidence of hemolysis as mentioned in the inclusion criteria. ${ }^{11}$

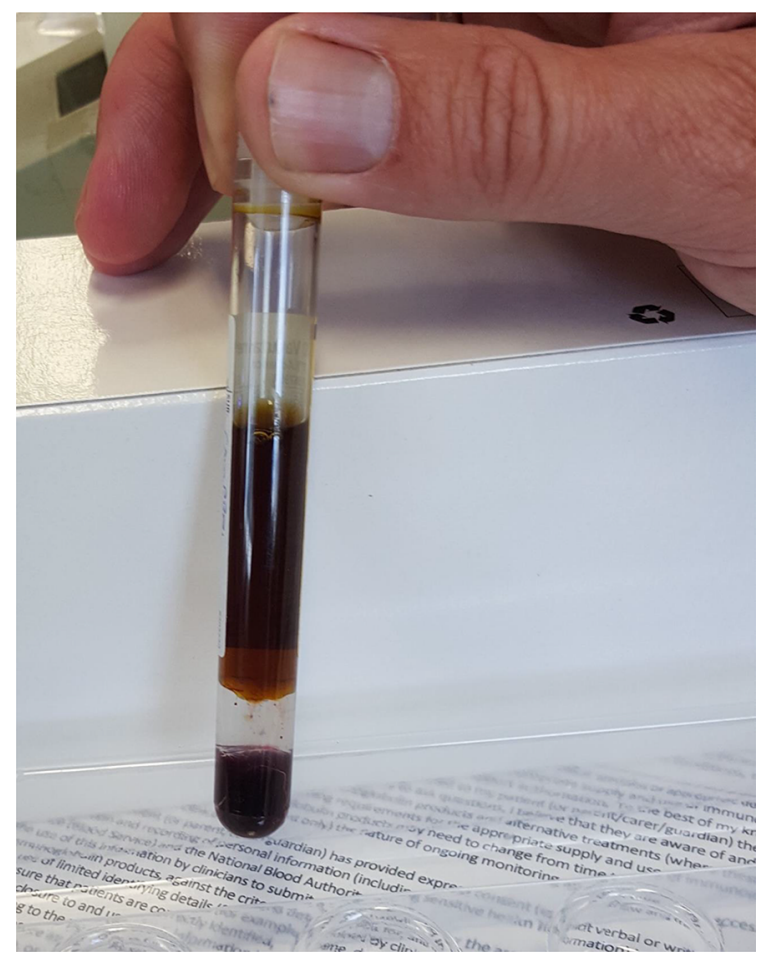

Figure I Hemoglobinemia (dark brown plasma) due to intravenous immunoglobulinmediated intravascular hemolysis. 


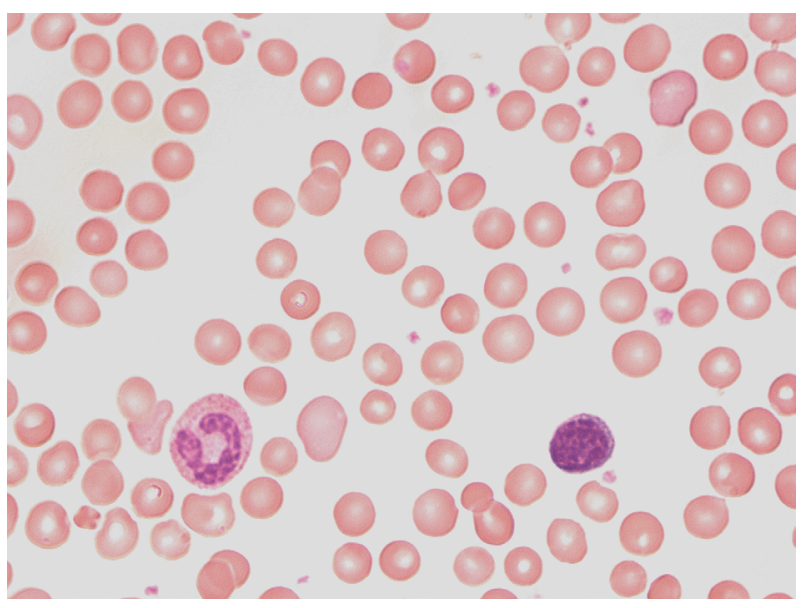

Figure 2 Increase in spherocytes due to intravenous immunoglobulin-mediated hemolysis (1000× magnification).

Pre-infusion matching of IVIG with recipients according to blood type is not feasible. Hence, administration of IVIG to group A, B, or AB recipients could potentially lead to HTR similar to a blood transfusion mismatch. Therefore, it is very important to implement measures to restrict the titers of the $\mathrm{ABO}$ antibodies contained in IVIG. ${ }^{4}$

\section{IVIG production}

IVIG products are manufactured from plasma separated from more than thousand donors - whole blood or plasmapheresis donors. Multiple stages are generally involved in the production of IVIG for plasma which includes serial precipitations, chromatographic purification, dedicated viral clearance steps, concentration, and addition of excipients to confer stability of IVIG products.

Cohn-Oncley fractionation and other manufacturing processes have multiple steps in the production of IVIG. There are many variations in different manufacturing methods. Changes in $\mathrm{pH}$, ionic strength, temperature, or alcohol and protein content during manufacturing can change the characteristics in the final IVIG product. Other factors that can alter the stability are mixing speeds, chromatographic methods, filters, buffer composition, and so on. Depending on the unique proprietary manufacturing process of IVIG, the final products will contain variable levels of components such as IgG subclasses, excipients, aggregates, other plasma proteins, and isoagglutinins. Hence, variations in the average isoagglutinin levels in IVIG could occur due to the differences in manufacturing methods of IVIG. ${ }^{10} \mathrm{ABO}$ isoagglutinins in plasma are mostly IgM class antibodies; however, IgG and IgA classes can also occur. In the fractionation and manufacturing processes involved in the production of IVIG,
IgG isoagglutinins are generally retained. Since the donor plasma pools contain a high proportion of group O plasma, IVIG products will contain anti-A, anti-B, and anti-A,B isoagglutinins. ${ }^{4}$

The methods that are used currently in the production of IVIG are listed below.

1. Cohn-like fractionation method involves multiple precipitation steps and purification, and the final product fraction (F)II contains IgG and coagulation factors. In these methods, isoagglutinins are precipitated in FIII at low $\mathrm{pH}$ of 5.4-6.3 and low-ionic-strength conditions, while most other IgG components are relatively soluble under such conditions (Figure 3). Apart from isoagglutinins, FIII also contains proenzymes and lipoproteins. ${ }^{12}$

2. Chromatography-based process was developed to achieve higher purity and yield of IVIG and hence rational utilization of limited resource of human plasma. This method involves a single precipitation step initially, followed by fractionation with octanoic

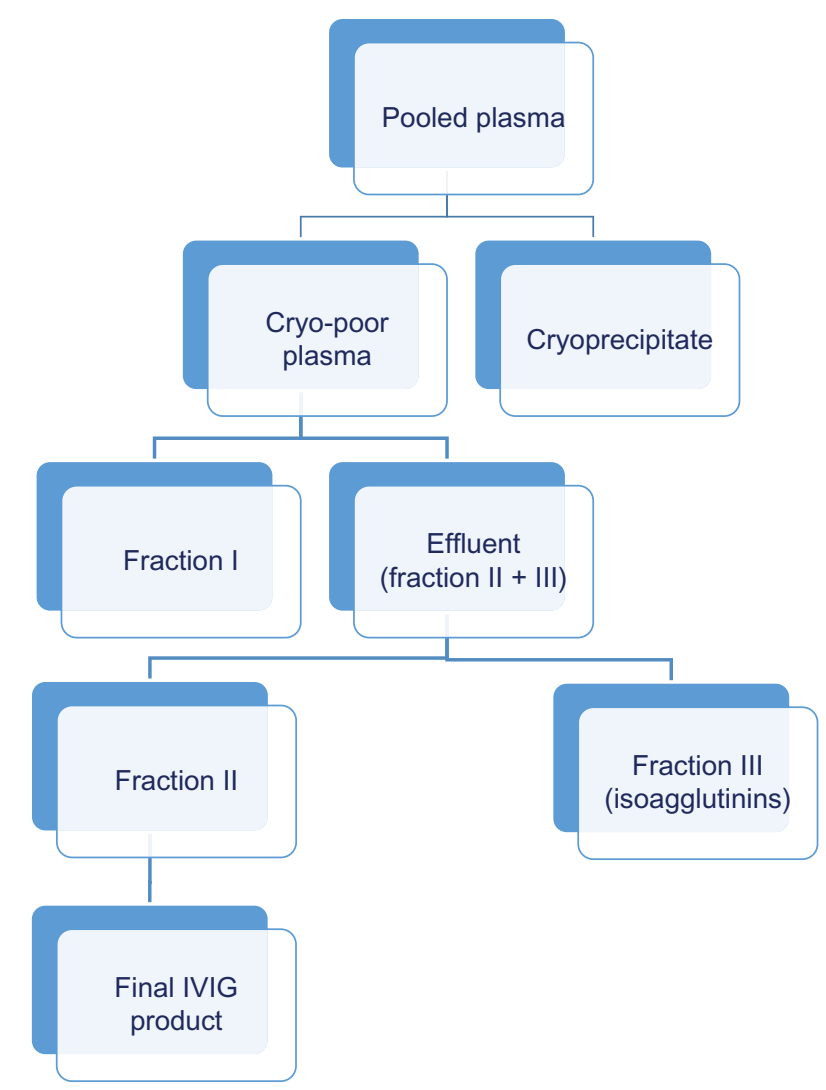

Figure 3 Schematic diagram of Cohn-like process for manufacture of IVIG from pooled plasma.

Note: Final IVIG product is produced from fraction II, and fraction III contains isoagglutinins.

Abbreviation: IVIG, intravenous immunoglobulin. 


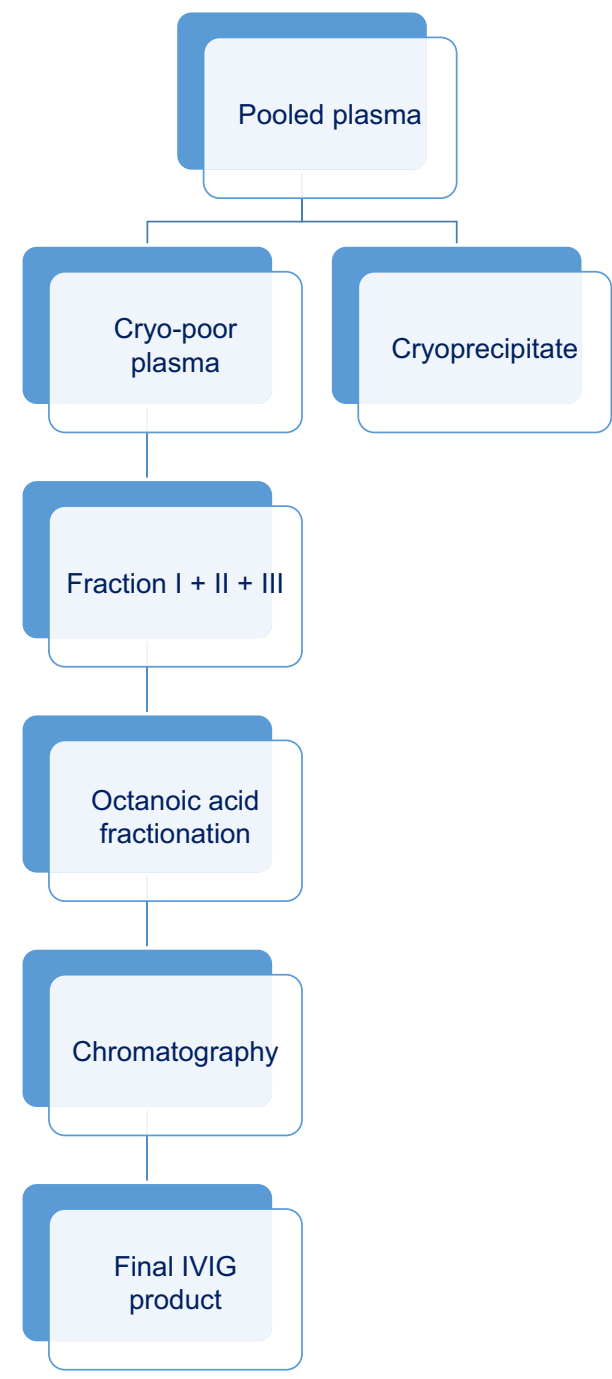

Figure 4 Schematic diagram of chromatography-based process of manufacture of IVIG from pooled plasma.

Abbreviation: IVIG, intravenous immunoglobulin.

acid and chromatography (Figure 4). When compared with Cohn-like fractionation method, chromatography-based IVIG products have generally lower concentrations of $\operatorname{IgA}$ and prekallikrein activator. ${ }^{13,14}$

Romberg et al ${ }^{12}$ compared the effectiveness of the two manufacturing processes, Cohn-like fractionation method and chromatography-based process, to reduce the levels of anti-A and anti-B isoagglutinins in IVIG products. They compared Sandoglobulin (CSL Behring AG, Berne, Switzerland), which is a lyophilized, sucrose-stabilized IVIG prepared by Cohn-like fractionation, with Privigen (CSL Behring $\mathrm{AG}$ ) which is $10 \% \mathrm{~L}$-proline stabilized IVIG produced by a chromatography-based process. The levels of anti-A and anti$B$ isoagglutinins were measured by the indirect agglutination test (IAT). For each fractionation step, the reduction in isoagglutinin levels was assessed in laboratory- and industry-scale experiments using IAT and a flow cytometry-based assay. The flow cytometry method involved fluorescence-activated cell sorting IgG-binding (FACS anti-A) and was used to determine the concentration of anti-A isoagglutinin in FII+III, FII, and the final IVIG product. Following incubation with A1 blood type RBCs, anti-A isoagglutinin level was calculated on the basis of relative median fluorescence intensity of $\mathrm{RBC}$-bound isoagglutinin. It was observed that the median anti-A isoagglutinin titer in 248 Sandoglobulin lots was 2, which was three titer steps lower than the median titer of 16 measured in 651 Privigen lots. Also a five-titer step isoagglutinin reduction was observed over the whole process from 512 to 16 in laboratory-scale Cohn-like fractionation. On the other hand, the chromatography-based manufacturing process did not result in the reduction of anti-A or anti-B isoagglutinin contents.

Hence, unlike Cohn-like fractionation processes, the chromatography-based IVIG manufacturing methods do not cause significant reduction of isoagglutinin levels. ${ }^{12}$ Manufacturing methods that do not have precipitation of IgG in the steps will result in higher levels of isoagglutinins and products of similar biophysical properties in the final product. Since those products have an increased risk of IVIGassociated hemolysis, dedicated isoagglutinin reduction steps are required to minimize the potential risk. ${ }^{15,16}$

\section{Risk factors and pathogenesis Risk factors}

The known risk factors for IVIG-associated hemolysis are

1. Administration of high-dose IVIG,

2. Non-O blood group recipients, and

3. Indication of inflammatory or autoimmune disorder. ${ }^{17}$

Blood donors of $\mathrm{O}$ group have anti-A and anti-B antibodies, A-group donors have anti-B antibodies, and B-group donors have anti-A antibodies. Blood donors of $\mathrm{AB}$ group do not have ABO antibodies in their blood. Hence, the plasma pool for IVIG production from a large number of donors would naturally contain antibodies against $\mathrm{A}$ and $\mathrm{B}$ antigens found in patients belonging to blood groups $\mathrm{A}, \mathrm{B}$, and $\mathrm{AB}{ }^{18}$ $\mathrm{O}$ blood group individuals can also produce isoagglutinins that react with both $\mathrm{A}$ and $\mathrm{B}$ antigens, known as anti-A,B, in addition to anti-A and anti-B isoagglutinins. These are antibodies of IgG class, are reported to cause clinically significant hemolysis and their titers are not routinely measured in IVIG products. ${ }^{4}$

Donors with excessive titers of $\mathrm{ABO}$ antibodies could potentially contribute to the high final quantity of $\mathrm{ABO}$ 
antibodies in IVIG products, which might eventually lead to hemolytic reactions. ${ }^{19}$ One percent of healthy donors can have antibodies against other common blood group (nonABO) antigens.

Patient characteristics are also deemed to play a significant part in IVIG-associated hemolysis, since significant clustering of cases of hemolysis is not seen among patients who have received the same batch of IVIG. The risk of hemolysis due to anti-A or anti-B antibodies in IVIG may depend on the recipient's secretor status. Soluble A and B antigens in the plasma which are present in secretors can cause neutralization of anti-A or anti-B antibodies in IVIG. Hence, blood group non-secretors have a higher risk of hemolysis due to the lack of soluble blood group antigens to neutralize anti-A or anti-B antibodies from IVIG. ${ }^{1}$ Other patient factors that may play a role in IVIG-associated hemolysis include Fc receptor polymorphisms, complement receptor polymorphisms, and antigen density on RBCs. Fc $\gamma$ receptor polymorphisms may play a role in susceptibility to IVIG-associated hemolysis. For example, polymorphisms of immunoglobulin receptor FcyRIIIa (CD16) on macrophages can determine the rapidity with which opsonized RBCs are removed from the circulation. ${ }^{20}$ Predisposition to IVIG-associated hemolysis might also be determined by associated comorbid medical conditions or medications that can cause hemolysis. ${ }^{17}$ It is observed from epidemiologic data that anti-A antibodies are most commonly involved in IVIG-associated hemolysis; other significant risk factors being patient- and productrelated factors. ${ }^{21}$

Even though higher IVIG doses are associated with hemolysis, the relationship between the doses of anti-A or anti-B isoagglutinin and IVIG-associated hemolysis remains uncertain. A prospective study on IVIG-associated hemolysis reporting rates before and after the addition of isoagglutinin depletion steps will help resolve this contradiction. ${ }^{22}$

Certain aspects associated with the manufacture of IVIG products may also increase the risk for hemolysis such as the type of excipient used in IVIG. In a study, relatively higher rates of IVIG-associated hemolysis were observed with amino acid (glycine, L-proline) - stabilized liquid IVIG products. ${ }^{23}$ Another study postulated that increased risk of hemolysis can occur due to increased dimerization in liquid preparations than in lyophilized preparations of IVIG. This increased risk of hemolysis may result from increase in complement fixation by dimerized anti-A and/ or anti-B adherent to RBCs. Also, IVIG preparations using chromatography-based processes are found to have higher anti-A and anti-B in the final products. ${ }^{24}$

\section{Mechanisms}

There are two mechanisms by which circulating RBCs are damaged by immune-mediated mechanism.

1. Intravascular destruction of RBCs by complementmediated lysis, which is mostly initiated by antibodies of IgM class. IgM bound to RBCs in the circulation mediates the activation of complement cascade which leads to the damage of red cell membrane causing intravascular hemolysis. The common red cell antibodies that cause intravascular hemolysis by complementmediated mechanism are anti-A and anti-B. Rarely other red cell antibodies such as anti-Jka, -Jkb, -Vel, and -Lea can lead to intravascular lysis. ${ }^{18}$ Intravascular hemolysis can cause massive hemolysis of $200 \mathrm{~mL}$ of RBCs which can lead to rapid drop of $\mathrm{Hb}$ by $5 \mathrm{~g} / \mathrm{dL}$ within hours. Hence, this can be potentially fatal if the patient is not promptly treated by compatible packed red cell transfusions. ${ }^{25}$

2. Extravascular destruction by immune cells, which detect RBC-bound IgG and complement. Extravascular hemolysis occurs in the mononuclear phagocyte system of predominantly the spleen and liver. ${ }^{26}$ Infection, inflammation, autoimmune hemolysis, or cytokine release states can contribute to hyperactivity of the macrophages. ${ }^{22}$

ABO isoagglutinins are mostly IgM antibodies, but $\mathrm{IgG}$ and $\operatorname{IgA}$ classes are also present in plasma. These have the potential of causing clinically significant hemolysis. Hence, when IVIG is administered if the recipient is group A, B, or AB, both IgM and IgG classes have the potential to activate complement and cause intravascular lysis. Rarely, IVIG-associated hemolysis occurs as a result of high-titer hemolysin activity. ${ }^{4}$

Since hemolytic complications occur in patients being treated for autoimmune/inflammatory conditions, rather than primary immunodeficiency, a two-hit hypothesis has been postulated for IVIG-associated hemolysis - the first hit being administration of high-dose IVIG in a non-O blood group patient and the second hit is the presence of an underlying inflammatory or highly sensitized state in the patient. The underlying inflammatory disease predisposes to the activation of splenic macrophages, which in turn increase their capacity for hemolysis of antibody or C3b-coated RBCs. For this hypothesis, a threshold effect has been suggested, with highest risk of hemolysis in patients with the most primed immune system and receiving high doses of IVIG. ${ }^{27}$ The preliminary results of a prospective study 
by Pendergrast et al supported the "two-hit" hypothesis proposed by Padmore. ${ }^{22}$

The mechanisms involved in IVIG-associated hemolysis are complex and include:

1. Pre-coating of RBCs due to underlying medical condition,

2. Transfer of high levels of alloantibodies to pre-coated RBCs,

3. Hemolysis triggered by passive transfer of $\operatorname{IgG}$ and binding to blood group antigens.

Antibodies other than blood group alloantibodies precoated to RBCs might also contribute to hemolysis in IVIG recipients. $^{5}$

\section{Challenges}

Due to the immunomodulatory potential of high-dose IVIG, its clinical indications have increased over time. ${ }^{6}$ Since 2011 , increased numbers of HTRs after IVIG treatment have been reported to the FDA from health care providers. ${ }^{10}$

To address the challenges and discuss the possible solutions for IVIG-associated hemolysis, a workshop was held in January 2014. ${ }^{28}$ Hematologists, transfusion medicine specialists and scientists, experts in epidemiology, IVIG manufacturing, and product testing, along with representatives of FDA and other international regulatory bodies attended the workshop. The objective of the workshop was to discuss epidemiology and product and patient risk factors of IVIG-associated hemolysis and to explore strategies to lower the incidence of clinically significant hemolysis in patients treated with IVIG products.

Many challenges and unmet needs were identified, and these were identified as potential areas for further research.

1. Better understanding of the pathogenesis of IVIGassociated hemolysis.

2. Identification of major patient risk factors and other key risk factors for IVIG-associated hemolysis.

3. To determine the IVIG product related risk factors for hemolysis and any other risk factors besides anti-A and/or anti-B levels.

4. To study the incidence of HTRs associated with newer indications for IVIG products.

5. Laboratory tests for patients and/or products that have predictive value for IVIG-associated hemolysis and whether there is need to revisit the current anti-A and anti-B testing techniques for IVIG products.
6. To establish the exact threshold isoagglutinin titers in IVIG products that causes hemolysis in the recipients.

7. Analyze evidence in favor of further reduction of antiA and anti-B titers in IVIG products.

8. Manufacturing processes that could effectively remove anti-A and/or anti-B from IVIG products.

9. Examine the evidence regarding cost-effectiveness to restrict donor plasma units with high titers of anti-A and/or anti-B from IVIG production.

10. In patients who require high doses of IVIG products, research regarding feasibility and effectiveness of administering IVIG products/lots with lower titers of anti-A or anti-B.

The proceedings and outcomes of the workshop were published as peer-reviewed articles in the Transfusion journal for the benefit of all the stakeholders, in particular clinicians prescribing IVIG and for scientists, hematologists, and transfusion medicine specialists who are generally consulted in the event of IVIG-associated hemolysis. ${ }^{10}$

\section{Epidemiology and burden of IVIG- associated hemolysis}

The exact incidence of IVIG-associated hemolysis will be difficult to determine, since majority of cases either go unnoticed or are not reported. Since most of the available data are based on spontaneously reported events of hemolysis and the fact that under-reporting is expected to be approximately $>80 \%$, there is absence of a proper denominator for the number of patients treated. ${ }^{29}$ The approximate incidence of IVIG-associated hemolysis was $1.6 \%$, reported in an adult case series. ${ }^{9}$ In contrast, a relatively high incidence of $16 \%$ is observed in younger patients with Kawasaki disease. ${ }^{30}$

Berg et $a{ }^{17}$ performed a retrospective qualitative analysis on HTRs associated with IVIG therapy in 263 patients for a 10 -year period from 2003 to 2012. These pharmacovigilance data represent the biggest database available currently for the study of cases of IVIG-associated hemolysis. For the study, a protocol was developed for use by four companies manufacturing IVIG products in order to collect and compile reports of all adverse effects of IVIG-associated hemolysis worldwide. The objectives of this study were to analyze potential risk factors of IVIG-associated hemolysis such as patient-related factors, indications of IVIG, dose levels ( $\mathrm{g} / \mathrm{kg}$ body weight and total doses) administered, hemagglutinin titers in different IVIG products, and identify other possible risk factors. Hemolysis was observed in all age groups 
(0-92 years) and the median age of patients at the time of hemolytic event was 51 years. Majority of patients with HTRs were A group (72\%) followed by AB group (18\%). Very few cases of HTRs were reported in O blood group, but these episodes were in the context of allogeneic stem cell transplant recipients with blood group mismatch. In this study, it was also observed that the frequency of IVIG-associated hemolysis in patients with $\mathrm{AB}$ blood group exceeds the frequency of patients with A blood group when compared to the prevalence of these blood groups in the general population; the ratio in relation to population was 2.6-9.0 for AB group when compared to 1.8-2.8 for the A group. The indications for use of IVIG were studied in patients who experienced hemolysis. Immune-mediated thrombocytopenia was the commonest indication (42\%) followed by Guillain-Barre syndrome (40\%). Other common indications were myasthenia gravis, chronic inflammatory demyelinating polyneuropathy, and Kawasaki disease. These five indications accounted for $58 \%$ of the patients treated with IVIG. ${ }^{17}$

A 3-year retrospective case series analyzed children treated for Kawasaki disease who developed IVIG-associated hemolytic anemia. It was observed that Kawasaki disease patients are at greater risk for HTRs due to the following factors: lower baseline $\mathrm{Hb}$ levels, underlying acute inflammatory condition, and oxygen requirements during acute illness. ${ }^{31}$ It was postulated that younger patients are at higher risk for IVIG-associated hemolysis, since RBCs from younger patients bind immune complexes more strongly than those of older patients. ${ }^{32}$

Regarding the timing of hemolytic episodes after IVIG administration, Winiecki et al observed that $55 \%$ of cases had hemolysis diagnosed within $48 \mathrm{~h}$ and $75 \%$ of cases within the first $96 \mathrm{~h}$ of the last infusion of IVIG. ${ }^{7}$ In Berg's study, approximately two-thirds of patients developed HTRs between 0 and $72 \mathrm{~h}$ after the last dose of IVIG, and the remaining one-third of cases were identified at a later period (72 h-14 days). ${ }^{17}$ Due to the lack of prospective data, it will be difficult to ascertain the time of onset of hemolysis. Hence, FDA recommends to check $\mathrm{Hb}$ in patients at higher risk of hemolysis prior to infusion and 36-96 h after IVIG infusion. ${ }^{21}$

In Berg's study, $58 \%$ of patients were reported to have received one or more units of $\mathrm{RBC}$ transfusions for the management of HTRs. In all patients who received a transfusion, starting $\mathrm{Hb}$ levels were compared with those who did not receive transfusions for hemolytic episodes. The mean starting $\mathrm{Hb}$ for non-transfused patients was $13.3 \mathrm{~g} / \mathrm{dL}$ versus $11.9 \mathrm{~g} / \mathrm{dL}$ for transfused patients $(p<0.001)$. Hence, patients with low $\mathrm{Hb}$ at the start of treatment have a greater risk of HTRs that require a transfusion. The mean $\mathrm{Hb}$ at the end of treatment was also compared for the two groups, and it was observed that for those requiring transfusion, $\mathrm{Hb}$ at the end of treatment was significantly lower $(7.2 \mathrm{~g} / \mathrm{dL})$ than for those who did not $(8.6 \mathrm{~g} / \mathrm{dL})(p<0.001)$. However, both transfused and non-transfused patients had similar reduction in $\mathrm{Hb}$ levels of $4.6 \mathrm{~g} / \mathrm{dL}(p=0.9){ }^{17}$

Padmore $^{27}$ analyzed the data collected from all the cases of possible IVIG-associated hemolysis reported in literature and identified 129 cases. He observed that $88 \%$ of the patients received high-dose IVIG, as defined by $\geq 1 \mathrm{~g} / \mathrm{kg}$ for 2 consecutive days or $2 \mathrm{~g} / \mathrm{kg}$ total dose or $\geq 100 \mathrm{~g}$ IVIG administered over $2-4$ days or $\geq 0.4-0.5 \mathrm{~g} / \mathrm{kg}$ for $4-7$ consecutive days. Most of the cases $(96 \%)$ were treated with IVIG for inflammatory or immune-mediated disorders. Majority of the adult cases $(97 \%)$ of IVIG-associated hemolysis occurred in non-O blood group patients, and among them, 73\% were blood group A patients, $10 \%$ were blood group $\mathrm{B}$, and $16 \%$ were blood group AB. Among the 22 pediatric cases $(<16$ years of age), $50 \%$ were group A, 14\% were group B, and $36 \%$ were group $\mathrm{AB}$. The study also analyzed the mean drop in $\mathrm{Hb}$. Pediatric cases were found to have a slightly larger mean drop of $\mathrm{Hb}$ compared with the adult mean. The largest mean reduction in $\mathrm{Hb}$ was seen in blood group $\mathrm{AB}$ cases, when compared to A and B blood group patients who had similar mean reductions in $\mathrm{Hb}^{27}$

Among the 263 cases in the study by Berg et al, ${ }^{17}$ information on lot number of the IVIG products was available in $52 \%$. Isoagglutinin titers (anti-A and anti-B) of each IVIG lot were originally determined by IAT by the manufacturers that participated in this study. The median anti-A and anti-B titers were 16 and 8 , respectively, in the IVIG lots associated with hemolytic episodes, and the maximum hemagglutinin titers were $<64$. Hemagglutinin dose is calculated by multiplication of the product titer and IVIG total dose administered (in grams). There was no significant difference in the percentage of patients receiving packed cell transfusions across the different hemagglutinin dose groups $(p=0.39) .{ }^{17}$

\section{Preventive measures}

Since HTRs associated with IVIG are usually due to ABO antibodies, reduction in the titers of these antibodies in IVIG should theoretically help to lower the frequency of hemolysis. ${ }^{18}$ Research in titers of ABO antibodies in blood products has provided information that assists in reducing the adverse effects of IVIG products. ${ }^{33}$ This will help manufacturers and 
regulatory agencies to limit the titers of anti-A and anti-B in the IVIG products in an attempt to lower the risk of hemolysis after IVIG therapy.

Different brands of IVIG vary in their average isoagglutinin content. Some of the current manufacturers produce IVIG products with higher retention of isoagglutinins. These differences are due to variations in the processes for removal of isoagglutinins. Studies are underway to identify methods to increase yield, lower production costs, and increase patient safety. ${ }^{10}$

The manufacturing methods that do not have precipitation steps will result in higher levels of isoagglutinins in the final IVIG product and therefore have an increased risk of IVIG-associated hemolysis. Hence, dedicated isoagglutinin reduction steps will be required to minimize the potential risk. ${ }^{15}$ Newer methods are being employed by the manufacturers to reduce anti-A and anti-B titers in IVIG products. In a study by Hoefferer et al, ${ }^{16}$ adsorption of alloantibodies by immune-affinity chromatography (IAC) was investigated and found to be an effective method for reduction in anti-A and anti-B titers, which was similar to those in cold-ethanol fractionation technique. Preliminary results in the study lots showed decrease in anti-A and anti-B titers by $\sim 90 \%$ and also a three-titer step decrease in isoagglutinin levels in the IAC modified IgG product compared to the unmodified product. Such processes that results in significant reduction of anti-A and anti-B titers can potentially minimize the risk of IVIGassociated hemolytic events in patients. ${ }^{16}$

Recently, IVIG manufacturers have developed beadbound oligosaccharides, which mimic $\mathrm{A}$ and $\mathrm{B}$ antigens and act as isoagglutinin removal resins. These resins are capable of removing $\sim 80 \%$ of isoagglutinin content in the IVIG products; however, these are not capable of removing isoagglutinin completely. The potential disadvantages of such isoagglutinin reduction procedures are removal of cross-reactive antibacterial or antiviral antibodies and hence reduction in the efficacy of IVIG in the management of patients with primary and secondary immunodeficiency disorders. ${ }^{21}$

Another strategy investigated to prevent hemolytic episodes is donor screening of anti-A by automated IAT and exclusion of donors with high titers from plasma pooling and fractionation. In a study it was observed that removal of donor plasma with high-titer isoagglutinin plasma units can lead to reduction in the levels of isoagglutinins by twofold. The disadvantage of this approach is that $5 \%-7 \%$ of donors will be excluded from blood/plasma donation for producing IVIG. ${ }^{34}$ In a cohort study of 310 O-group donors, isoagglutinin titers were measured and high-titer donors were excluded before plasma pooling for producing IVIG. By this method, a twofold reduction of isoagglutinin levels was expected; however, it was observed that exclusion of hightiter donations actually did not have a significant impact due to dilution effects. Approximately $80 \%$ of O-group donors have antibody titers $<1,000$. Since only a small proportion of O-group donors have antibody titers $>1,000$, the effects of high-titer antibodies in the plasma pool are minimal. ${ }^{35}$

Based on the available evidence, national regulatory bodies recommend testing of anti-A and anti-B titers in commercial IVIG products, and their titers should be kept $<64$. $^{5}$ European Pharmacopoeia also recommends maximum titers of 64 for anti-A and anti-B for 5\% (w/v) IVIG products. ${ }^{1}$ Despite meeting these requirements, hemolysis can occur in patients administered with IVIG products.

Another preventive measure is that IVIG recipients should be monitored closely for signs and symptoms of hemolysis. A few studies observed that majority of cases experienced hemolysis within $96 \mathrm{~h}$ after the last dose of IVIG. ${ }^{7,17}$ IVIG-associated hemolysis can be prevented by monitoring $\mathrm{Hb}$ when administering high dose IVIG to patients with non-O blood group for treatment of inflammatory or immune-mediated disorder. In patients with IVIG-associated hemolysis requiring packed cell transfusion, O-group packed RBCs should be preferably transfused to avoid further hemolysis due to anti-A and/or anti-B antibodies. ${ }^{27}$ Based on the observations, it is recommended to monitor $\mathrm{Hb}$ before IVIG infusion and 36-96 h post-infusion in patients at higher risk of hemolysis and low baseline $\mathrm{Hb}$. FDA has approved labeling for some IVIG products that recommends checking $\mathrm{Hb}$ in patients at higher risk of hemolysis before infusion and 36-96 $\mathrm{h}$ after IVIG infusion. ${ }^{21}$

Spontaneous reporting of IVIG-related adverse events and analysis are essential tools in the quality assurance for drug safety, which also help to understand and prevent such episodes. The data essential in the reporting process are lot numbers of IVIG products, dosage ( $\mathrm{g} / \mathrm{kg}$ body weight), preinfusion $\mathrm{Hb}$ values, indications of IVIG treatment, co-morbid illness, and concomitant medications. This will help to have a better understanding of the risk factors involved in IVIGassociated hemolysis. Health care professionals should be encouraged to report adverse events to the manufacturers or regulatory bodies. ${ }^{17}$

A few management options that are suggested for the prevention of hemolysis include IVIG dosing based on lean body weight and premedication with steroids. Splitting the 
total dose of IVIG to multiple doses over a longer period of time has been postulated for the prevention of hemolysis. However, these are not evidence based..$^{21}$ Other important steps in the prevention of IVIG-associated hemolysis are education of physicians and health care providers about this risk and to create awareness on its prevention and management.

On the basis of current evidence, many changes in the manufacturing process, confirmatory testing, and quality assurance systems have been instituted to assess and reduce the titers of isoagglutinins, thereby reducing the risk of hemolysis. Continued vigilance is also necessary to ensure that such changes in manufacturing methods do not cause any adverse events in the patients. ${ }^{10}$

\section{Management of IVIG-associated hemolysis}

Currently, there are no quality data or guidelines for the management of IVIG-associated hemolysis. Based on the analysis of cases in the literature, Desborough et al proposed the following steps for management of patients with IVIGassociated hemolysis. ${ }^{1}$

\section{Patient management}

1. Cessation of IVIG infusion.

2. Monitor vital signs and urine output and supportive management for the patient.

3. Proper hydration and/or RBC transfusions if necessary

a. Since IVIG-associated hemolysis is usually a selflimiting condition, red cell transfusion should be avoided if possible.

b. Packed RBCs for transfusions should preferably be $\mathrm{O}$ group.

4. Acute renal failure is a potential complication. If conservative management of renal impairment fails, nephrology review should be sought for consideration of renal replacement therapy.

5. In the event of severe IVIG-associated hemolysis, IVIG products should be avoided in future and alternate management options should be considered.

6. If re-treatment with IVIG is essential, a careful riskbenefit assessment should be performed.

There were a few reports of management of hemolysis with corticosteroids and/or plasma exchange. Also switching to another IVIG product may be helpful if the patients require IVIG in the future. However, the risk of subsequent recurrence of clinically significant hemolysis is unclear. ${ }^{36}$

\section{Laboratory investigations and reporting}

1. Perform laboratory tests for hemolysis.

2. DAT and antibody specificity.

3. Check titers of ABO-group antibodies in the IVIG product infused. If IVIG product contains high titer for anti-A or anti-B antibodies, quarantine of the IVIG batch/lot should be considered.

4. Reporting the adverse event to regulatory/vigilance body.

\section{Summary and future directions}

Hemolysis caused by IVIG is usually self-limited, and a small proportion may be clinically significant. Patients at risk of IVIG-associated hemolysis are non-O blood group recipients who receive high-dose IVIG for inflammatory or autoimmune disorder. In the recent years, FDA received increased reports of hemolytic episodes after IVIG treatment. This is due to the fact that the clinical indications and usage of high-dose IVIG have increased over time. Clinically significant hemolysis associated with IVIG administration has been identified as a major problem requiring special attention.

Close monitoring of $\mathrm{Hb}$ level in high-dose IVIG recipients, especially in those with low $\mathrm{Hb}$, can assist in early detection of hemolysis and prevent serious complications. On the basis of available evidence, many changes in the manufacturing process, confirmatory testing, and quality assurance systems have been introduced to assess and minimize the risk of hemolysis. Continued vigilance is also necessary to ensure that such changes in manufacturing methods do not cause any adverse events in the patients.

The workshop based on IVIG-associated hemolysis discussed various aspects such as epidemiology, patient risk factors, product risk factors, challenges, and the possible solutions. It also helped to identify avenues of future research for prevention or reduction of hemolysis in IVIG recipients.

1. Methods to determine the exact incidence of clinically significant IVIG-associated hemolysis, such as better voluntary reporting tools, patient registry, etc.

2. Exploration into the possible risk factors of hemolysis, other than anti-A and anti-B agglutinins.

3. Studies of pathogenesis of inflammatory process that are deemed to contribute to IVIG-associated hemolysis and the inflammatory markers that could be used as a predictor for hemolysis. 
4. Investigation of better in vitro assays of hemolytic potential of IVIG products, that is, hemagglutination activity.

5. Methods to improve the precision and reproducibility of hemagglutinin tests, such as use of flow cytometry or other methods.

6. Investigation of the constituents of IVIG which can influence the hemolytic potential in different IVIG products such as anti-A,B isoagglutinin content, antibody subclass, or antibody glycosylation.

7. Investigations of certain management practices to prevent recurrences in patients who developed IVIGassociated hemolysis such as

a. premedication with corticosteroids,

b. use of ideal body weight rather than actual body weight for dosing,

c. different split dosing regimens for high doses of IVIG, and

d. pre-infusion in vitro hemolysin test to check compatibility, that is, patient RBCs incubated with specific IVIG to be infused. ${ }^{21}$

\section{Disclosure}

The author reports no conflicts of interest in this work.

\section{References}

1. Desborough MJ, Miller J, Thorpe SJ, Murphy MF, Misbah SA. Intravenous immunoglobulin-induced haemolysis: a case report and review of the literature. Transfus Med. 2014;24(4):219-226.

2. Orange JS, Hossny EM, Weiler CR, et al. Use of intravenous immunoglobulin in human disease: a review of evidence by members of the Primary Immunodeficiency Committee of the American Academy of Allergy, Asthma and Immunology. J Allergy Clin Immunol. 2006;117 (4 Suppl):S525-S553.

3. Case J, Schiff P, Walsh J. Blood-group antibodies in human immune serum globulin. N Engl J Med. 1972;287(7):359-360.

4. Branch DR. Anti-A and anti-B: what are they and where do they come from? Transfusion. 2015;55(Suppl 2):S74-S79.

5. Spath PJ, Granata G, La Marra F, Kuijpers TW, Quinti I. On the dark side of therapies with immunoglobulin concentrates: the adverse events. Front Immunol. 2015;6:11.

6. Sewell WA, Kerr J, Behr-Gross ME, Peter HH; Kreuth Ig Working Group. European consensus proposal for immunoglobulin therapies. Eur J Immunol. 2014;44(8):2207-2214.

7. Winiecki S, Baer B, Chege W, et al. Complementary use of passive surveillance and Mini-Sentinel to better characterize hemolysis after immune globulin. Transfusion. 2015;55(Suppl 2):S28-S35.

8. Bussel JB, Eldor A, Kelton JG, et al. IGIV-C, a novel intravenous immunoglobulin: evaluation of safety, efficacy, mechanisms of action, and impact on quality of life. Thromb Haemost. 2004;91(4):771-778.

9. Daw Z, Padmore R, Neurath D, et al. Hemolytic transfusion reactions after administration of intravenous immune (gamma) globulin: a case series analysis. Transfusion. 2008;48(8):1598-1601.

10. Scott DE, Epstein JS. Hemolytic adverse events with immune globulin products: product factors and patient risks. Transfusion. 2015;55 (Suppl 2):S2-S5.
11. Taylor E, Vu D, Legare C, Keene D. Intravenous immune globulinrelated hemolysis: comparing two different methods for case assessment. Transfusion. 2015;55(Suppl 2):S23-S27.

12. Romberg V, Hoefferer L, El Menyawi I. Effects of the manufacturing process on the anti-A isoagglutinin titers in intravenous immunoglobulin products. Transfusion. 2015;55(Suppl 2):S105-S109.

13. Ballow M, Berger M, Bonilla FA, et al. Pharmacokinetics and tolerability of a new intravenous immunoglobulin preparation, IGIV-C, $10 \%$ (Gamunex, 10\%). Vox Sang. 2003;84(3):202-210.

14. Teschner W, Butterweck HA, Auer W, et al. A new liquid, intravenous immunoglobulin product (IGIV $10 \%$ ) highly purified by a state-of-theart process. Vox Sang. 2007;92(1):42-55.

15. Salvatore A, Esin S, Batoni G, Ascione E, Farina C, Nardini C. Anti-A and anti-B hemagglutinin depletion during Cohn purification process of 5\% immunoglobulin. Transfusion. 2015;55(Suppl 2):S110-S116.

16. Hoefferer L, Glauser I, Gaida A, et al. Isoagglutinin reduction by a dedicated immunoaffinity chromatography step in the manufacturing process of human immunoglobulin products. Transfusion. 2015;55(Suppl 2): S117-S121.

17. Berg R, Shebl A, Kimber MC, Abraham M, Schreiber GB. Hemolytic events associated with intravenous immune globulin therapy: a qualitative analysis of 263 cases reported to four manufacturers between 2003 and 2012. Transfusion. 2015;55(Suppl 2):S36-S46.

18. Flegel WA. Pathogenesis and mechanisms of antibody-mediated hemolysis. Transfusion. 2015;55(Suppl 2):S47-S58.

19. Pierce RN, Reich LM, Mayer K. Hemolysis following platelet transfusions from ABO-incompatible donors. Transfusion. 1985;25(1):60-62.

20. Kumpel BM, De Haas M, Koene HR, Van De Winkel JG, Goodrick MJ. Clearance of red cells by monoclonal IgG3 anti-D in vivo is affected by the VF polymorphism of Fcgamma RIIIa (CD16). Clin Exp Immunol. 2003;132(1):81-86.

21. Scott DE, Epstein JS. Safeguarding immune globulin recipients against hemolysis: what do we know and where do we go? Transfusion. 2015;55(Suppl 2):S122-S126.

22. Pendergrast J, Willie-Ramharack K, Sampson L, Laroche V, Branch DR. The role of inflammation in intravenous immune globulin-mediated hemolysis. Transfusion. 2015;55(Suppl 2):S65-S73.

23. Dantal J. Intravenous immunoglobulins: in-depth review of excipients and acute kidney injury risk. Am J Nephrol. 2013;38(4):275-284.

24. Kahwaji J, Barker E, Pepkowitz S, et al. Acute hemolysis after high-dose intravenous immunoglobulin therapy in highly HLA sensitized patients. Clin J Am Soc Nephrol. 2009;4(12):1993-1997.

25. Mohamed M, Bates G, Eastley B. Massive intravascular haemolysis after high dose intravenous immunoglobulin therapy. Br J Haematol. 2013;160(5):570.

26. Garratty G. The James Blundell Award Lecture 2007: do we really understand immune red cell destruction? Transfus Med. 2008;18(6):321-334.

27. Padmore R. Possible mechanisms for intravenous immunoglobulinassociated hemolysis: clues obtained from review of clinical case reports. Transfusion. 2015;55(Suppl 2):S59-S64.

28. Chtourou S. Efficient methods in place to reduce anti-A/anti-B isoagglutinin titers in immunoglobulin products. FDA/PPTA/NHLBI Public Workshop-Strategies to Address Hemolytic Complications of Immune Globulin Infusions. US Food and Drug Administration, Silver Spring (MD). Last updated March 102014.

29. Hazell L, Shakir SA. Under-reporting of adverse drug reactions: a systematic review. Drug Saf. 2006;29(5):385-396.

30. Berard R, Whittemore B, Scuccimarri R. Hemolytic anemia following intravenous immunoglobulin therapy in patients treated for Kawasaki disease: a report of 4 cases. Pediatr Rheumatol Online J. 2012;10(1):10.

31. Luban NL, Wong EC, Henrich Lobo R, Pary P, Duke S. Intravenous immunoglobulin-related hemolysis in patients treated for Kawasaki disease. Transfusion. 2015;55(Suppl 2):S90-S94.

32. Shapiro S, Kohn D, Miller B, Gershon H. Erythrocytes from young but not elderly donors can bind and degrade immune complex- and antibody-bound C3 in vitro. Clin Exp Immunol. 1994;95(1):181-190. 
33. Fontaine MJ, Mills AM, Weiss S, Hong WJ, Viele M, Goodnough LT. How we treat: risk mitigation for ABO-incompatible plasma in plateletpheresis products. Transfusion. 2012;52(10):2081-2085.

34. Siani B, Willimann K, Wymann S, Marques AA, Widmer E. Isoagglutinin reduction in human immunoglobulin products by donor screening. Biol Ther. 2014;4(1-2):15-26.
35. McVey J, Baker D, Parti R, Berg R, Gudino M, Teschner W. Anti-A and anti-B titers in donor plasma, plasma pools, and immunoglobulin final products. Transfusion. 2015;55(Suppl 2):S98-S104.

36. Azizi G, Abolhassani H, Asgardoon MH, et al. Managing patients with side effects and adverse events to immunoglobulin therapy. Expert Rev Clin Pharmacol. 2016;9(1):91-102.
International Journal of Clinical Transfusion Medicine is an international, peer-reviewed, open access, online journal publishing clinicalexperimental, policy-making and evidence-based practices of all topics pertaining to clinical transfusion medicine. Original research, short reports, reviews, case reports and commentaries are invited
The manuscript management system is completely online and includes a very quick and fair peer-review system, which is all easy to use. Visit http://www.dovepress.com/testimonials.php to read real quotes from published authors.

Submit your manuscript here: https://www.dovepress.com/international-journal-of-clinical-transfusion-medicine-journal 